\title{
Absorption Components in the Nucleus of NGC 3227
}

\author{
Dawei $\mathrm{Xu}^{1,2}$, Stefanie Komossa ${ }^{1}$, Vadim Burwitz ${ }^{1}$, Peter Predehl ${ }^{1}$ \\ ${ }^{1}$ Max-Plank-Institut für extraterrestrische Physik, Giessenbachstrasse, \\ D-85748 Garching, Germany \\ ${ }^{2}$ National Astronomical Observatories, Beijing 100012, China
}

\begin{abstract}
X-ray spectroscopy provides a wealth of information about the properties of the surrounding reprocessing material of AGN. We present results from a $96 \mathrm{ks}$ exposure of NGC 3227, taken with the Low Energy Transmission Grating Spectrometer on board the Chandra Xray Observatory. A partial covering model fits well the resulting X-ray spectrum. We investigate the dusty nuclear environment of this galaxy, and in particular concentrate on its warm absorber.
\end{abstract}

\section{The X-ray view of NGC 3227}

NGC 3227 is a nearby Seyfert type 1.5 galaxy at a distance of $18 \mathrm{Mpc}$. It has been intensely studied across the electromagnetic spectrum (see Komossa \& Fink 1997 for a review). In X-rays, the source is highly variable, suggesting the dominance of AGN-type emission. The combination of optical, UV and X-ray data led to the suggestion of a dusty warm absorber in NGC 3227.

We present a Chandra observation of NGC 3227, performed with the LETGS (first results of this study were presented by Komossa et al. 2001). These data allow to address a number of important questions, for instance: test of the dusty warm absorber model, a search for extended emission from a starburst component, and a search for emission from 'hot' gas spatially coincident with the NLR. Here, we concentrate on the absorption spectrum.

\section{Observation and data analysis}

NGC 3227 was observed as part of the guaranteed time observer program, with the LETGS in combination with HRC-S. The observation was carried out on October 23-24, 2000 with an effective exposure time of $96 \mathrm{ks}$.

Spectral analysis: Simple powerlaw fits are unacceptable and leave two kinds of residuals: an absorption-edge-like feature around $\sim 0.7 \mathrm{keV}$, and a similar feature above $1 \mathrm{keV}$. These features can be accounted for by the presence of a warm absorber, and a partial coverer, respectively. The X-ray spectrum of NGC 3227 (Fig. 1) is best described by a warm-absorbed powerlaw of which about $10 \%$ is seen directly, whereas the remainder of the intrinsic continuum is absorbed by material with a column density of $N_{\mathrm{H}} \approx 3.110^{22} \mathrm{~cm}^{-2}$. 
Temporal Variability: We find NGC 3227 is highly variable as also seen in previous X-ray observations of this galaxy. In Fig. 1 we show the observed count rate as a function of time using $1000 \mathrm{~s}$ bins. The highest amplitude of variability is a factor $\sim 5$ within $96 \mathrm{ks}$.
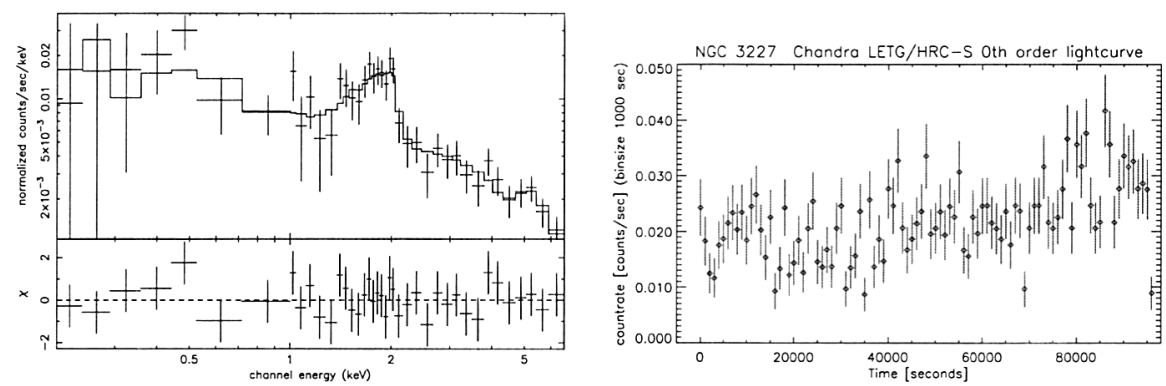

Figure 1. Left: Chandra LETGS spectrum of NGC 3227. The solid line is the best-fit WA plus partial covering model. Right: Background subtracted X-ray lightcurve - Chandra LETGS zeroth order signal with 1000 s binsize.

\section{Absorption components and their variability}

As first pointed out by Komossa et al. (2001), the absence of a huge amount of cold absorption in an HST-UV spectrum of NGC 3227 taken $~ 8.5$ months prior to our Chandra observation (Crenshaw et al. 2001) suggests that the cold absorption is variable on the time scale of months or less, and could be due to a BLR cloud passing our line-of-sight. On the other hand, some (but not all) previous observations of NGC 3227 also favored a partial covering model: see Reichert et al. 1985 (two Einstein SSS observations) and George et al. 1998 (one out of two $A S C A$ observations). These observations suggest variable obscuration is frequent in this galaxy. In this context, it is interesting to note that Risaliti et al. (2002) find variable absorption columns of cold material in almost all the sources of their sample of Seyfert 2 galaxies. The same may hold for intermediate-type Seyferts like NGC 3227.

\section{References}

Crenshaw, D.M., et al. 2001, ApJ, 555, 63

George, I.M., et al. 1998, ApJ, 509, 146

Komossa, S., \& Fink H. 1997, A\&A, 327, 483

Komossa, S., Burwitz, V., Predehl, P., \& Kaastra, J. 2001, in: The Central Kiloparsec of Starbursts and AGN, ASP Conf. ser., 249, 411

Reichert, G.A., Mushotzky, R.F., Petre, R., \& Holt, S.S. 1985, ApJ, 296, 69

Risaliti, G., Elvis, M., \& Nicastro, F. 2002, ApJ, 571, 234 